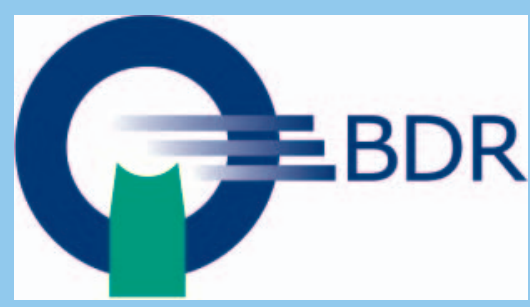

\section{Nachwuchsarbeit im BDR}

Die Arbeit des BDR - wie auch die der DRG - ist nur möglich, da sich die Mitglieder, neben ihren Mitgliedsbeiträgen, auch persönlich engagieren. In AGs zur Gebührenordnung, Hygiene, Weiterbildungsordnung und anderen, teilweise temporären Arbeitsfeldern. Eines davon ist im BDR zukünftig die Nachwuchsarbeit. „Früher“ fanden junge Radiolog:innen, die in Praxen einstiegen, den Weg direkt zum BDR. Dies ist schon seit einiger Zeit kein selbstverständlicher Weg. Aber angestellte Radiolog:innen, Weiterbildungsassistent:innen, junge Praxisinhaber:innen und Klinik-Radiolog:innen haben (auch) einen festen Platz in Berufsverbänden.

Im BDR gibt es nun eine „NachwuchsBeauftragte“. Diese Position, die dem Vorstand kooptiert ist, wird von Frau Dr. Ulrike Engelmayer wahrgenommen. Über ihre Motivation berichtet sie im nachfolgenden Text.

Sabine Lingelbach, Geschäftsführerin

\section{Warum ich mich im BDR engagiere}

Ich möchte unternehmerische Verantwortung übernehmen und meinen Arbeitsplatz aktiv mitgestalten! Dieser Wunsch reifte in mir, als ich 2015 nach mehreren Jahren als Fachärztin in einem Haus der Schwerpunktversorgung anfing, nach einer Praxis zu suchen, die eine leistungsstarke Gemeinschaft von freiberuflichen Radiolog:innen bildet und in die ich mich als Gesellschafterin einbringen kann. Dass diese Suche 6 Jahre dauern würde, hätte ich damals nicht gedacht. Aber nun ist - endlich! - mein Einstieg als Gesellschafterin in eine Gemeinschaftspraxis in greifbarer Nähe.
Während meiner Suche wurde mir bewusst, wie wichtig die gute Vernetzung von uns Radiolog:innen für eine erfolgreiche Praxisführung ist. Unser Fachgebiet ist heiß umkämpft, da die finanziellen Ressourcen für uns Radiolog:innen knapper werden und andere Fachgruppen und fachfremde Wirtschaftsunternehmen in unseren Fachbereich drängen. Ich bin überzeugt, dass ich mich als selbstständige Radiologin im ambulanten Sektor nur behaupten kann, wenn ich mich mit anderen Radiolog:innen zusammenschließe, um Synergien zu nutzen und um gemeinsam für unsere berufsständischen, fachlichen und wirtschaftlichen Interessen einzutreten. Deswegen bin ich Mitglied im BDR geworden.

Die Radiologie hat eine Schlüsselstellung in der modernen Medizin und gewährleistet die genaue Auswahl und Vorhaltung optimaler Diagnose- und Therapieverfahren unter Einhaltung höchster medizinischer und apparativer Qualitätsstandards. Da die radiologische Diagnostik in der wohnortnahen und sektorenübergreifenden Versorgung der Bevölkerung unverzichtbar ist, ist es essenziell, dass wir Radiolog:innen uns in der ärztlichen Selbstverwaltung einbringen, als Partner der politischen Organe und der Kostenträger auftreten, das Abrechnungs- und Gebührenwesen mitgestalten und unsere Belange in Zusammenarbeit mit anderen Berufsverbänden und Fachgesellschaften vertreten.

Die Versorgung unserer Patient:innen wird nur gut und umfassend bleiben, wenn in unseren Praxen auch weiterhin flexibles, innovatives und engagiertes Arbeiten möglich ist und interessante und lukrative Zukunftsperspektiven in Bezug auf Arbeitsplatzsicherheit, Verdienst und die fachli- chen und persönlichen Entfaltungsmöglichkeiten aller Mitarbeitenden geboten werden. Damit dies gewährleistet bleibt, muss die Führung radiologischer Praxen in den Händen von freiberuflichen Ärzt:innen liegen. Hierfür brauchen wir NachwuchsRadiolog:innen, die den Schritt in die Selbstständigkeit wagen und das unternehmerische Risiko auf sich nehmen. Für sie möchte ich mich im Rahmen meines berufspolitischen Engagements ganz besonders einsetzen.

Mir ist es daher ein großes Anliegen, die Themen zu adressieren, die für Radiolog:innen im Übertritt vom stationären in den ambulanten Sektor und an der Schwelle zur Selbstständigkeit entscheidend sind. Dazu zähle ich neben den neuen fachlichen Anforderungen, denen man im ambulanten Sektor begegnet, auch die Herausforderungen in Bezug auf Management und Unternehmens- und Mitarbeiterführung in einer Praxis. Außerdem möchte ich, zusammen mit den erfahrenen Kolleg:innen im Berufsverband, Wege finden, wie wir jüngeren Radiolog:innen, angesichts des zunehmenden Einflusses von großen, zum Teil international agierenden Kapitalgebern und Konzernen, uns in der ambulanten Versorgung erfolgreiche Zukunftsperspektiven aufbauen können.

Mit diesen Themen möchte ich mich im BDR einbringen und so Kolleg:innen für das Engagement im BDR und für die Niederlassung in einer radiologischen Praxis begeistern.

\section{Dr. Ulrike Engelmayer \\ Friedberg}

